\title{
Forestación piloto con tara en Cajamarca
}

(1) Oswaldo Rojas Lazo

(2) Nora Rojas Pérez

${ }^{(3)}$ Germán Díaz Chuquiruna

\begin{abstract}
RESUMEN
En los últimos años, se ha despertado el interés por el cultivo de la tara aplicando técnicas agroforestales adecuadas en vías de incrementar su productividad, pues en su hábitat la tara crece en forma silvestre. Este interés se debe a que del fruto de la tara, tanto de la vaina como de las semillas, se obtienen múltiples productos de aplicación industrial.

El Perú es actualmente el primer productor y exportador mundial de los derivados de la tara.

Este proyecto piloto de forestación con tara se lleva a cabo en el departamento de Cajamarca (años 2008 al 2009), distrito de San Juan, caserío de Cachilgón, cuya población cuenta con $40 \%$ de adultos analfabetos quienes se dedican a la agricultura de productos de pan llevar y se apoyan económicamente con la recolección de la vaina de tara que crece en forma silvestre. Para demostrar a los pobladores la facilidad del manejo agroforestal de la tara y su rentabilidad económica, se viene desarrollando el proyecto piloto de forestación con 1546 plantones de tara, requiriendo una inversión inicial de 12234 soles, un costo anual referencial de 4720 soles y recuperando toda la inversión en el año 2012
\end{abstract}

Palabras clave: Forestación piloto con tara, forestación con tara Cajamarca..

\section{CAJAMARCA PILOT FORESTY WITH TARA}

\section{ABSTRACT}

In the last years, because of the variety of industrial applications of the tara's fruit (the shell and the seeds can be processed to obtain a lot of products), the interest on cultivate tara using correct techniques is enhanced.

This project of forestation with tara is carried out in Cajamarca Department (years 2008-2009), San Juan district, Cachilgon country house, which population has $40 \%$ of adults without instruction. They are dedicated to the foodstuffs agriculture and earn money with recolectation of the fruit of the tara, where the tara grows as a wild tree. To show to the population the facility of handling the agriculture of the tara and its economical profitability, it has being develop this pilot project of forestation with 1546 tree of tara, which requires an initial investment of 12243 peruvian soles, an annual referential cost of 4720 peruvian soles and recover all the investment in 2012.

Key words: Pilot forestation with tara, forestation with tara Cajamarca (Peru).

\section{INTRODUCCIÓN}

El árbol de la tara (CAESALPINIA SPINOSA) es una leguminosa que crece en las cuencas del Pacífico y del Atlántico. Su cultivo es aprovechable económicamente cuando crece en un rango de 800 a $3100 \mathrm{msnm}$. La tara es un árbol que resiste a las plagas y enfermedades; para subsistir, necesita poca agua y para una óptima producción, requiere de 400 a $600 \mathrm{~mm}$ de lluvia anual. Los frutos se cosechan a partir del cuarto año en un promedio de 20 a $40 \mathrm{~kg}$ por cosecha, por árbol. Este rendimiento puede mejorar con un manejo agroforestal tecnificado, pudiéndose cosechar hasta dos veces por año. Los frutos de la tara son recolectados y comercializados por los lugareños a los acopiadores locales.

Del fruto de la tara, es posible obtener taninos (de la vaina), gomas, hidrocoloides y galactómanos (de la semilla), los cuales se utilizan como aditivos o materia prima en muchas industrias; e incluso, aunque en menor proporción, actualmente se aplica con fines medicinales y en proceso de teñidos (en la época prehispánica se usaba en la medicina folclórica y en el proceso de teñidos).

Desde hace 10 años, estos productos son comercializados en los mercados internacionales, siendo el Perú el primer productor y exportador mundial de los derivados de la vaina de la tara, y China el mercado más importante. En la costa del Perú, se encuentran las empresas procesadoras y exportadoras de los productos de la vaina de la tara.

El precio referencial en dólares FOB de un kilogramo del polvo de la vaina de tara es de 1,33 y de la goma en polvo de la semilla de tara 3,06 dólares. La creciente demanda actual en los mercados internacionales, exige a los productores agrícolas ofertar productos de calidad y si se cultivan mediante manejo orgánico, se pueden obtener mejores precios.

La microcuenca de San Juan se encuentra ubicada al sureste de la ciudad capital del departamento de Cajamarca, a una altitud referencial de $2225 \mathrm{msnm}$, tiene una extensión de 69,66 $\mathrm{km}^{2}$, cuenta con una población de 5224 habitantes $(40 \%$ de

1. Ingeniero Industrial. Profesor del Departamento de Diseño y Tecnología Industrial - UNMSM. E-mail: orojasla@homail.com

2. Lic. en Química. Profesora del Departamento de Fisicoquímica - UNMSM E-mail: norarpc@hotmail.com

3. Ingeniero Industrial.

E-mail: daiz1313@hotmail.com 
adultos analfabetos) que se dedican a la agricultura no tecnificada de productos de panllevar y se apoyan económicamente con la venta de la vaina de tara que crece en forma silvestre. Estas plantas silvestres cuando son tiernas, son consumidas por los animales, y los árboles maduros sirven como madera para consumo doméstico.

La característica topográfica de la microcuenca es de una quebrada, en la que se encuentran cerca de 1500 hectáreas de tierras en secano que están aptas para ser forestadas con plantones de tara.

En el volumen $10, N .^{\circ} 2$ de la revista de investigación Industrial Data se publicó un primer artículo "La tara y condiciones de reforestación en el Alto Jequetepeque, Microcuenca de San Juan-Cajamarca", en dicho artículo se trata sobre la planta de la tara, se presenta un diagnóstico situacional de la microcuenca de San Juan.

Por su importancia económica, y debido a que el cultivo de la tara no requiere suelos ni topografía especial, se está realizando un proyecto piloto de forestación con tara a fin de incentivar su cultivo aplicando un manejo agroforestal adecuado. Este proyecto piloto se está desarrollando en el caserío de Cachilgón con 1546 plantones, con una inversión inicial de 12234 soles, un costo anual referencial de 4720 soles y se recuperará toda la inversión en el año 2012.

En el primer año se han perdido 343 plantones por diversos factores (22\% del total). La resiembra se rea- lizó en la época de lluvia. Los riegos se realizan por inundación y acarreo. Los abonos y los foliares se aplicaron a las plantas en la época de invierno, asimismo el control fitosanitario es constante. A diciembre de 2009 , han florecido 11 plantones $(0.7 \%$ del total).

Las experiencias de este proyecto piloto serán transmitidas a los pobladores mediante exposiciones orales y la elaboración de una guía didáctica, creando de esta manera una cultura agroempresarial en el campesinado.

En el último año, las municipalidades y ONGs están impulsando forestaciones de diversos tipos de plantas cultivadas en viveros. El aprovechamiento del canon minero en actividades productivas agroindustriales podría mejorar la situación socioeconómica de los pobladores.

En esta publicación, se abarcará el estudio de la forestación de los años 2008 y 2009.

\section{PREFERENCIAS RESPECTO A LA FORESTA- CION EN EL DISTRITO DE SAN JUAN}

En el cuadro 1, se presentan los precios de venta de la producción anual por hectárea de algunos productos que se producen en el distrito de San Juan. La tuna como medio de producción de la cochinilla, es la más rentable seguida por la tara.

En el cuadro 2, se presenta una encuesta realizada en el año 2007 sobre las preferencias de los habitantes del distrito de San Juan respecto a la fo-

Cuadro 1: Precios de venta promedio de la producción anual/ha, de la chirimoya, lúcuma, palta, tara y tuna

\begin{tabular}{|c|c|c|c|c|c|c|}
\hline Plantación & Riego & $\begin{array}{c}\text { Plantones } \\
\text { por ha }\end{array}$ & $\begin{array}{c}\text { Inicio de } \\
\text { Producc. (años) }\end{array}$ & $\begin{array}{l}\text { Producc. } \\
\text { tn/ha/año }\end{array}$ & $\begin{array}{c}\text { Precio } \\
\text { unitario tn } \mathrm{S} / .\end{array}$ & $\begin{array}{c}\text { Venta bruta } \\
\text { Anual S/. }\end{array}$ \\
\hline Chirimoya & sí & 320 & 5 & $6-11$ & 2000 & 22000 \\
\hline Lúcuma & sí & 320 & 5 & $5-10$ & 6000 & 60000 \\
\hline Palta & sí & 320 & 5 & $1-8$ & 2500 & 20000 \\
\hline Tara & no & 625 & 4 & $5-31$ & 2000 & 62000 \\
\hline Tuna fruto & no & 2500 & 3 & $4-30$ & 1000 & 30000 \\
\hline Tuna cochinilla & no & 2500 & 3 & $3-7$ & 30000 & 210000 \\
\hline Total & & & & & & 404000 \\
\hline
\end{tabular}

Fuente: Ministerio de Agricultura y lugareños año 2009.

Cuadro 2: Preferencia de los habitantes para la forestación del distrito de San Juan

\begin{tabular}{|c|c|c|c|c|c|c|c|}
\hline Edades & Chirimoya & Lúcuma & Palta & Tara & Tuna & otros & Total \\
\hline $14-29$ & 25 & 22 & 19 & 32 & 20 & 2 & 120 \\
\hline $30-45$ & 19 & 21 & 28 & 39 & 10 & 3 & 120 \\
\hline $46-61$ & 22 & 24 & 20 & 36 & 14 & 4 & 120 \\
\hline 62 -a más & 30 & 19 & 22 & 31 & 11 & 7 & 120 \\
\hline Total & 96 & 86 & 89 & 138 & 55 & 16 & 480 \\
\hline
\end{tabular}

Fuente: Elaboración propia (junio de 2007). 
restación. Se puede observar que la mayoría, sin distingo de edades prefieren forestar con tara.

\section{MERCADO DE LA TARA}

El Perú tiene muchos bosques naturales de tara, mientras que en los países vecinos (Bolivia, Chile, Ecuador, Venezuela y Colombia) no se tienen estadísticas de producción. A nivel nacional (cuadro 3), en los últimos años, el departamento de Cajamarca ha sido el primer productor.

En el cuadro 4, se presenta el proyecto corredor económico productivo forestal de Cajamarca de la institución civil "FONDEBOSQUE"; para el periodo 2007-2011; se ha proyectado reforestar 165000 ha, con plantaciones de pino, eucalipto, tara y agroforestería, designando 10000 ha $(6,07 \%)$ para plantaciones de tara.

En la Región de Cajamarca, los productores y recolectores comercializan la vaina de la tara a los acopiadores locales en costales de yute y/o polietileno con un peso, aproximado, de 46 a 50 kilogramos por costal (1 quintal). Los mayoristas con camiones trasladan a los centros de procesamiento que se encuentran en la costa del Perú.

De acuerdo al cuadro 5, la micrcocuenca de San Pablo es la que tiene mayor producción de tara seguida por el Alto Jequetepeque (Comprende los distritos de: Asunción, Chetilla, Magdalena y San Juan).

En el cuadro 6, se muestran los precios de la vaina de tara en chacra, las variaciones del precio es en función a la oferta siendo el motivo principal las temporadas de lluvias.
La empresa SOUTH AMÉRICA TANNIN CORPORATION ha sido la empresa que más exportó al mercado de China en el año 2009 (cuadro 7).

\section{CONTEXTO DEL PROYECTO PILOTO}

El proyecto piloto se desarrolla en el caserío de Cachilgón-Calaní (gráfico 1, plano de ubicación; gráfico 2, vista panorámica de Cachilgón-Calaní), al sur del distrito de San Juan, provincia y departamento de Cajamarca; se encuentra entre los 1800 y 2600 msnm; en la margen izquierda de río San Juan a 7 $\mathrm{km}$. de la carretera de la ciudad de San Juan, presenta un piso ecológico y agrícola (tierras fértiles y eriazas) apto para realizar la plantación de tara, existe mano de obra no calificada.

El caserío cuenta con una población de 290 habitantes (INEI-Censo de Población y Vivienda 2007). Comprende tierras de tenencia privada, el riego en los meses de diciembre a marzo es por aguas de lluvia y en época de sequía con las aguas del río o

Figura 1: Ubicación del proyecto de forestación

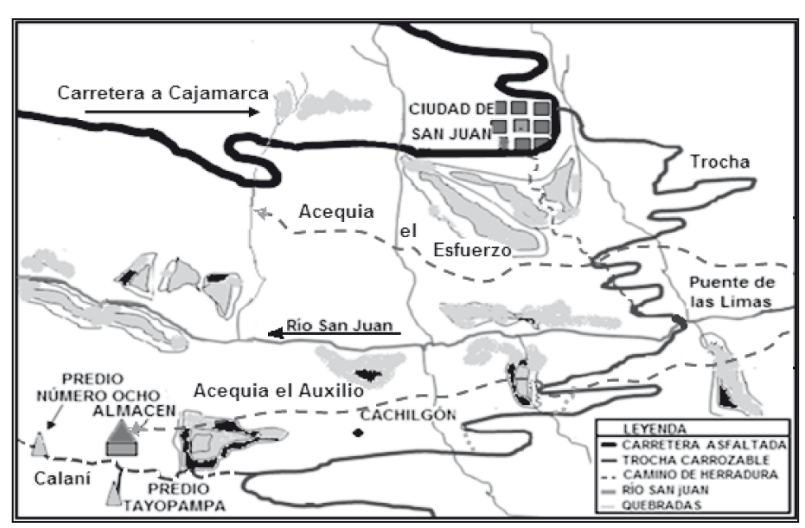

Fuente: Elaboración propia.

Cuadro 3: Departamentos productores de tara en toneladas (1997-2007)

\begin{tabular}{|l|c|c|c|c|c|c|}
\hline Departamentos & $\begin{array}{c}\text { Acumulado } \\
\mathbf{1 9 9 7} \mathbf{a} \mathbf{2 0 0 0}\end{array}$ & $\begin{array}{c}\text { Acumulado } \\
\mathbf{2 0 0 1} \mathbf{a} \mathbf{2 0 0 4}\end{array}$ & $\begin{array}{c}\text { Acumulado } \\
\mathbf{2 0 0 5 - 2 0 0 6}\end{array}$ & $\mathbf{2 0 0 7}$ & $\mathbf{\%}$ & Total \\
\hline Cajamarca & 4478 & 18174 & 17532 & 12514 & 42,0 & 52698 \\
\hline Ayacucho & 6503 & 8189 & 5591 & 1900 & 17,7 & 22183 \\
\hline Áncash & 2438 & 2556 & 3893 & 2054 & 8,6 & 10941 \\
\hline La Libertad & 589 & 2595 & 7227 & -- & 8,3 & 10411 \\
\hline Lambayeque & 64 & 5902 & 1479 & 4181 & 9,2 & 11626 \\
\hline Piura & 105 & 1593 & 2930 & -- & 3,7 & 4628 \\
\hline Otros & 1414 & 4259 & 3619 & 3868 & 10,5 & 13160 \\
\hline Total Nacional & 15591 & 43268 & 32271 & 24517 & 100,0 & 125647 \\
\hline
\end{tabular}

Fuente: (Consultor: Barriga R. Cesar, 2009).

http://www.sinia.minam.gob.pe/admElemento.php?accion=bajar\&idDocAdjunto=107

http://www.monografias.com/trabajos47/plantacion-de-tara/plantacion-de-tara2.shtml (abril 2009). 
Cuadro 4: Metas quinquenales de plantaciones 2007- 2011

\begin{tabular}{|c|c|c|c|c|c|c|}
\hline \multirow{2}{*}{ Sistema por cultivo } & \multicolumn{4}{|c|}{ Corredor económico productivo } & \multirow{2}{*}{$\begin{array}{c}\text { Superficie } \\
\text { ha }\end{array}$} & \multirow{2}{*}{$\%$} \\
\hline & Norte 1 & Norte 2 & Centro & Sur & & \\
\hline Plantación de pino & 15000 & 10000 & 15000 & 10000 & 50000 & 30,30 \\
\hline Plantación de eucalipto & 25000 & 25000 & 30000 & 20000 & 100000 & 60,60 \\
\hline Plantación de tara & 3000 & 2000 & 3000 & 2000 & 10000 & 6,07 \\
\hline Agroforestería & 1500 & 1250 & 1250 & 100 & 5000 & 3,03 \\
\hline Total & 44500 & 38250 & 49250 & 33000 & 165000 & 100,00 \\
\hline
\end{tabular}

Fuente: http://www.fondebosque.org.pe (abril 2009)

Cuadro 5: Producción de tara por microcuencas (Cajamarca), 2004

\begin{tabular}{|l|c|c|c|}
\hline \multirow{2}{*}{ Microcuencas } & \multicolumn{3}{c|}{ Año: 2004 } \\
\cline { 2 - 4 } & ha & Producción & Potenciales \\
\hline Alto Jequetepeque & 272 & 653 & 18500 \\
\hline Celendín & 180 & 432 & 26000 \\
\hline Chota & 138 & 331 & 10000 \\
\hline Contumazá & 272 & 653 & 39000 \\
\hline Jaén & 120 & 288 & 70000 \\
\hline Jesús & 236 & 566 & 5500 \\
\hline San Marcos & 230 & 552 & 28900 \\
\hline San Pablo & 298 & 715 & 34000 \\
\hline Total & 1746 & 3817 & 231900 \\
\hline
\end{tabular}

Fuente:http://www.regioncajamarca.gob.pe/descargas/deseco/05_09_01_PLAN_ESTRATEGICO_REGIONAL_ EXPORTADOR.pdf (mayo 2009).

de manantiales conducidos por 5 canales. Las vías de comunicación terrestre son: un camino de herradura y una trocha carrozable de Choropampa, San Juan y Asunción; funciona una Institución Educativa estatal de Inicial y Primaria, la mayoría de las tierras están forestadas por plantaciones silvestres de tara, chirimoya, motemote, nísperos, cansabocas, nogales, guarangos, paucos, guayabas, guabos, saraqueros, carrizos, pastos y otras especies vegetales.

El clima (temperatura promedio es de $16,4^{\circ} \mathrm{C}$, humedad relativa varia entre $44 \%$ y $66 \% \mathrm{http}: / / \mathrm{www}$. scribd.com/doc/19961127/PROYECTOS2007-68) es favorable para el crecimiento y producción de especies vegetales y animales, la agricultura y la ganadería que practican los lugareños es tradicional, realizan actividades agrícolas de sembríos menores estacionales (periodo de lluvia), siendo su economía de subsistencia.
Cuadro 6: Precio de la vaina de tara en la chacra (Cajamarca)

\begin{tabular}{|r|r|r|}
\hline \multirow{2}{*}{ Años } & \multicolumn{2}{|c|}{ Precio } \\
\cline { 2 - 3 } & \multicolumn{1}{|c|}{ (Soles/kg) } & (Soles/quintal) \\
\hline 1990 & 0.33 & 10 a 15 \\
\hline 1995 & 0.50 & 23 \\
\hline 2000 & 0.76 & 35 \\
\hline 2001 & 1.31 & 60 \\
\hline 2002 & 2.61 & 90 a 120 \\
\hline 2003 & 1.53 & 71 \\
\hline 2004 & 1.63 & 75 \\
\hline 2005 & 1.69 & 78 \\
\hline 2006 & 1 á 1.50 & 46 a 69 \\
\hline 2007 & 2.50 & 96 \\
\hline 2008 & 4.00 a 3.00 & 184 a 138 \\
\hline 2009 & 2.00 a 1.50 & 92 a 69 \\
\hline
\end{tabular}


El proyecto se viene desarrollando en las propiedades de la familia Díaz Chuquiruna, la mano de obra e inversión será proporcionada por la familia (2 hombres de 48 y 50 años; 3 mujeres de 80, 48 y 45 años, y 3 niños de 16, 14 y 7 años, que apoyan los fines de semana). Para casos especiales, se contratará personal adicional.

Para el proyecto, la familia Díaz tiene disponible 7 predios (cuadro 8); de ellos, 2 fueron seleccionados para el proyecto piloto teniendo en cuenta las variables involucradas en el cuadro 9.

Figura 2: precipitaciones mensuales promedio (mm), año 2003, en la microcuenca de San Juan, Alto Jequetepeque - Cajamarca

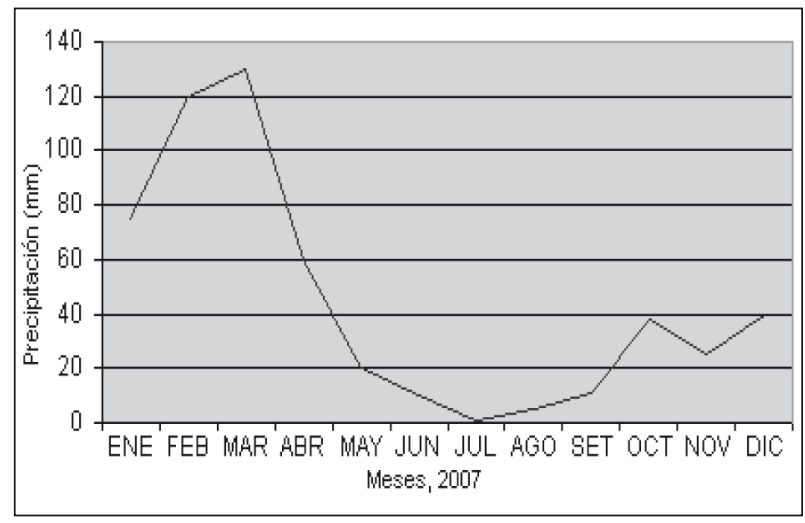

Fuente: http://www.condesan.org/cuencasandinas/jequetepeque.htm (enero 2009).
La cantidad de almácigos que se podía conseguir era de un máximo de 1800 unidades (cuota proporcionada por la Municipalidad de San Juan y posibilidad económica). Por hectárea, se pueden plantar entre 700 a 800 plantas. Las variables consideradas para seleccionar los predios piloto son: área, tenencia, comunicación, distancia a la ciudad, riego, seguridad y pendiente. La ponderación de los predios se detalla en el cuadro 8 .

Todos los predios tienen sus entradas y salidas independientes, el traslado en vehículo motorizado del último paradero (La capilla en Calaní) a la carretera de la ciudad de San Juan es de 35 minutos para un recorrido de $7,0 \mathrm{~km}$, mientras a pie por el camino de herradura, la distancia es de $5 \mathrm{~km}$ que se realiza en un estimado de 1 hora.

Para la puntuación se tomó como base 10 puntos para todas las variables. De acuerdo al cuadro 9, se seleccionaron a los predios Tayopampa con 53 puntos y el Número Ocho con 48 puntos, en ellos se realizará el proyecto piloto de forestación con tara.

En los predios seleccionados se venía sembrando productos de pan llevar y algunos árboles frutales, obteniéndose una rentabilidad anual promedio en cada uno de los predios de 200 soles.

Los dos predios seleccionados (cuadro 10) suman una área de 20000 m2 (2 ha), para una plantación

Cuadro 7: Empresas exportadoras de los derivados de tara y sus respectivos mercados.

\begin{tabular}{|l|c|c|c|c|}
\hline EMPRESAS EXPORTADORAS & MERCADOS & US \$. FOB 2007 & US \$. FOB 2008 & US \$. FOB 2009 \\
\hline $\begin{array}{l}\text { SOUTH AMÉRICA TANNIN } \\
\text { CORPORATION }\end{array}$ & China & & & 5332,04 \\
\hline SILVATEAM PERU SAC. & $\begin{array}{c}\text { Italia } \\
\text { China }\end{array}$ & 3248,05 & 4182,30 & \\
\hline EXPORTADORA EL SOL SAC. & $\begin{array}{c}\text { Argentina } \\
\text { Italia }\end{array}$ & 2217,92 & 4149,39 & 1352,22 \\
\hline MOLINOS CHIPOCO EIRL. & $\begin{array}{c}\text { Brasil } \\
\text { Bélgica }\end{array}$ & 1970,32 & 1710,68 & 1128,75 \\
\hline PRODUCTOS DEL PAÍS SA. & $\begin{array}{c}\text { China } \\
\text { Brasil } \\
\text { Países Bajos }\end{array}$ & 1014,72 & 3856,64 & 523,80 \\
\hline OTRAS EMRESAS & $\begin{array}{c}\text { Otros países } \\
\text { TOTAL }\end{array}$ & 5412,02 & 9446,35 & 4622,90 \\
\hline
\end{tabular}

Fuente: http://www.siicex.gob.pe/siicex/portal5ES.asp?_page_=172.17100\&_portletid_=sfichaproductoinit\&scriptdo=cc_fp_init\&pproducto=192\&pnomproducto= Tara (mayo 2009) 
Cuadro 8: Variables de cada predio

\begin{tabular}{|l|r|l|c|c|c|c|c|}
\hline \multirow{2}{*}{ Predios } & \multicolumn{7}{|c|}{ Descripción } \\
\cline { 2 - 8 } & Área $\mathbf{m}^{2}$ & Tenencia & Comunicación & $\begin{array}{c}\text { Distancia } \\
\text { a la ciudad }\end{array}$ & Riego & Seguridad & Pendiente \\
\hline Cacho & 5000 & título & rápida & $5,2 \mathrm{~km}$ & sí & sí & $0,0 \%$ \\
\hline Higuerón & 5400 & título & rápida & $5,1 \mathrm{~km}$ & sí & sí & $40 \%$ \\
\hline N. ${ }^{\circ}$ Ocho & 8500 & posesión & rápida & $5,4 \mathrm{~km}$ & no & no & $20-40 \%$ \\
\hline Quishuar & 4800 & título & muy rápida & $5,0 \mathrm{~km}$ & no & no & $45 \%$ \\
\hline Huanga & 12500 & posesión & lenta & $6,0 \mathrm{~km}$ & no & no & $50 \%$ \\
\hline La Julia & 6000 & título & rápida & $5,1 \mathrm{~km}$ & sí & sí & $30 \%$ \\
\hline Tayopampa & 11500 & título & rápida & $5,1 \mathrm{~km}$ & sí & & sí \\
\hline
\end{tabular}

Fuente: Elaboración propia.

Cuadro 9: Selección de predios agrícolas con ponderaciones

\begin{tabular}{|l|c|c|c|c|c|c|c|c|}
\hline \multirow{2}{*}{ Predios } & \multicolumn{7}{c|}{ Ponderaciones } & Total \\
\cline { 2 - 10 } & A & T & C & D & R & S & m & \\
\hline Cacho & 5 & 8 & 8 & 4 & 8 & 4 & 10 & 47 \\
\hline Higuerón & 3 & 8 & 8 & 4 & 6 & 5 & 5 & 39 \\
\hline Número Ocho & 8 & 5 & 8 & 5 & 6 & 8 & 8 & 48 \\
\hline Quishuar & 3 & 8 & 10 & 8 & 4 & 2 & 5 & 40 \\
\hline Huanga & 10 & 5 & 4 & 2 & 4 & 2 & 6 & 33 \\
\hline La Julia & 6 & 8 & 8 & 4 & 8 & 6 & 6 & 44 \\
\hline Tayopampa & 9 & 10 & 8 & 4 & 8 & 8 & 6 & 53 \\
\hline
\end{tabular}

A: área, T: tenencia, C: comunicación, D: distancia, R: riego, S: Seguridad y m: pendiente Fuente: Elaboración propia.

Cuadro 10: Predios pilotos para la plantación de tara

\begin{tabular}{|l|c|c|c|}
\hline \multicolumn{1}{|c|}{ Predios } & Tayopampa & Número Ocho & Total \\
\hline ÁREA DEL PREDIO & $11500 \mathrm{~m} 2$ & $8500 \mathrm{~m} 2$ & $20000 \mathrm{~m} 2$ \\
ALTITUD & $2200 \mathrm{msnm}$ & $2050 \mathrm{msnm}$. & -- \\
DISTANCIAS ENTRE PLANTAS & $4 \mathrm{~m} \times 4 \mathrm{~m}$ & $3,50 \mathrm{~m} \times 3,50 \mathrm{~m}$ & --- \\
PLANTAS TRASPLANTADAS & 735 plantones & 811 plantones & 1546 plantones \\
ÁREA REFORESTADA: & $11500 \mathrm{~m} 2$ & $8500 \mathrm{~m} 2$ & $20000 \mathrm{~m} 2$ \\
\hline NOTA: En 2 ha, se transplantarán 1546 plantones de tara. & \\
\hline
\end{tabular}

Fuente: Elaboración propia. 
de 1546 plantones de tara, la plantación se realizó en el mes de enero del 2008 aprovechando el agua de la lluvia (invierno).

En el gráfico 3, se muestra la zona de Calaní donde están ubicados los predios del proyecto piloto (la distancia entre los dos predios es de $2 \mathrm{~km}$ ). El predio Toyopampa es regado por el agua de la acequia proveniente del río Pacachar y el predio Número 8 es secano.

Figura 3: Predios seleccionados para el proyecto piloto

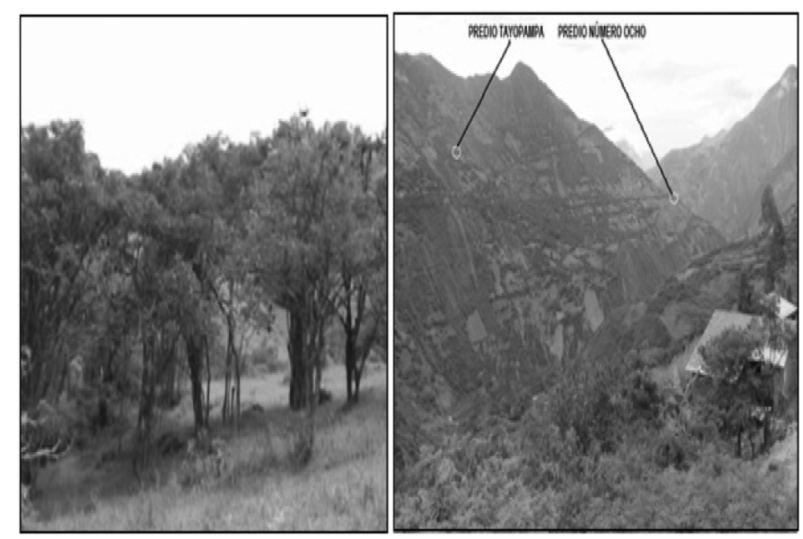

Fuente: Elaboración propia.

La tara se propaga por medio de semillas, se seleccionan de plantas con mejor producción (30 kgcosecha/árbol) y buen tamaño de vaina, se prepara un sustrato que es embolsado y remojado colocándose luego dos semillas hidratadas en el sustrato; se forman los almácigos en los viveros respectivos durante 4 a 6 meses para después ser transplantados al predio seleccionado. En el gráfico 4, se presenta un almácigo del vivero del distrito de San Juan listo para ser trasplantado (altura promedio de $20 \mathrm{~cm}$ ).

Para la distribución de los hoyos, se tienen dos formas, una es matricial rectangular y la otra de alineación inclinada formando triángulos equiláteros (gráfico 5). En el predio de Toyopampa por ser de riego y presentar topografía uniforme se utilizó el método matricial cuadrado (735 plantones) y en el predio Número Ocho por presentar ondulaciones y lomadas se usó el método de triángulos equiláteros (811 plantones).

La preparación del terreno consistió en el cercado del terreno, limpieza de piedras, extracción de malas hierbas, limpieza de acequias. La excavación de los hoyos fue de forma cilíndrica $(40 \mathrm{~cm}$ de diámetro, y $40 \mathrm{~cm}$ de profundidad). El pre abonado
Figura 4: Almácigos de tara listos para transplante (vivero San Juan)

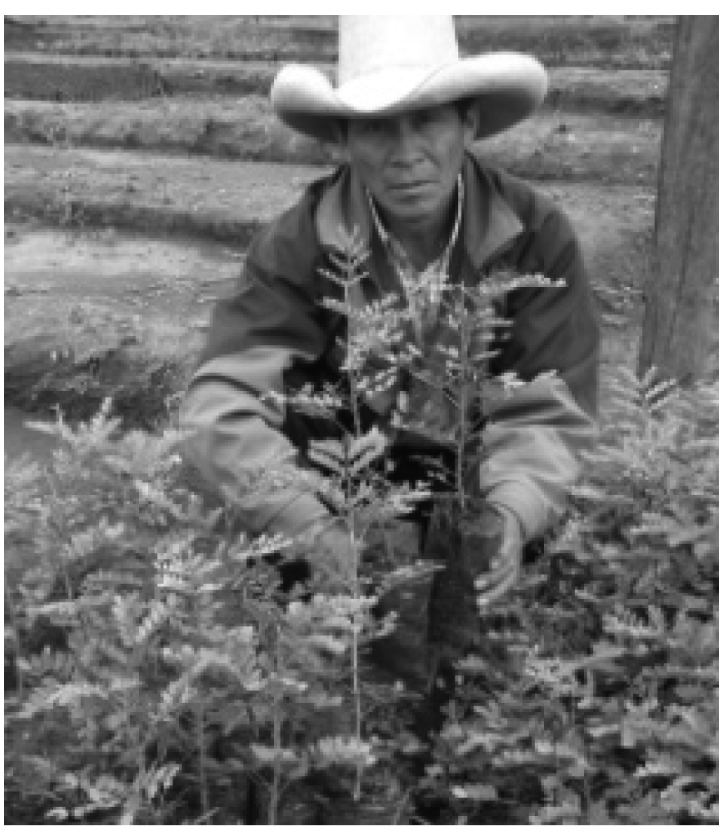

Fuente: Elaboración propia.

consistió en colocar $100 \mathrm{~g}$ de abono, (preparado con gallinaza $-50 \mathrm{~g}$ y excremento seco de ganado vacuno, lanar y caprino $-50 \mathrm{~g}$ ), encima $10 \mathrm{~cm}$ de tierra fértil.

Los plantones para el predio Número Ocho (811 plantones, 6 meses de crecimiento, altura promedio de $20 \mathrm{~cm}$ ) se adquirieron del vivero municipal del distrito de San Juan y, para el predio de Tayopampa (735 plantones, 4 meses de crecimiento, altura promedio $16.8 \mathrm{~cm}$ ), se adquirieron del vivero $1 \mathrm{de}$ Mayo.

El trasplante se programó para inicios de la época de lluvias (diciembre-marzo), En los hoyos ya abonados, se introducen los plantones con su respectivo sustrato (sin la bolsa de plástico), se fija con tierra

Figura N. ${ }^{5}$ : Separación entre plantones

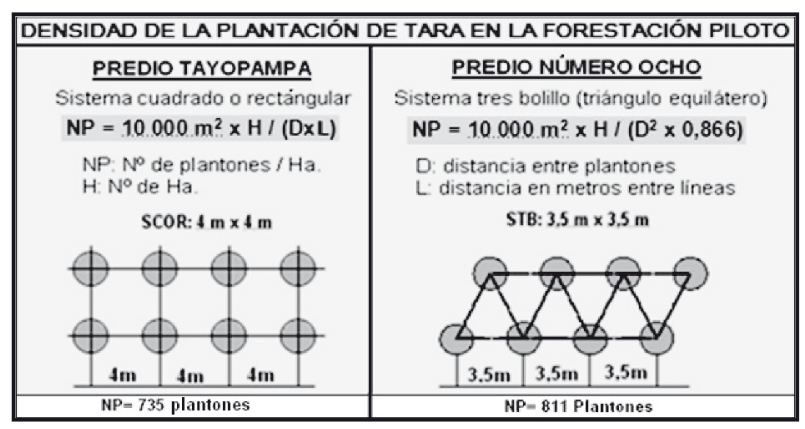

Fuente: Elaboración propia. 
dejando una altura de $10 \mathrm{~cm}$ para regar, al no haber lluvias se utilizó agua traída en bidones de plástico, se aplicó un litro por plantón (predio secano).

Para el 26 de diciembre de 2008, se tenían 343 plantones muertos, que representa el $22 \%$ de la plantación total. La mortalidad de los plantones fue a consecuencia de las inundaciones por las lluvias del mes de marzo (28\%), riego inadecuado (20\%), destrozo de hojas por la hormiga arriera (50\%) y pastoreo en los predios forestados (2\%). Los 343 plantones se resembraron en ambos predios en el mes de enero de 2009 aprovechando las lluvias estacionales. Al finalizar el año 2009, se tenían 62 plantones muertos en ambos predios (50 de la siembra de enero 2008 y 12 de la resiembra de enero de 2009, estos se resembraron en enero de 2010).

El abono durante los 2 primeros años de manejo forestal (2008-2009) estuvo compuesto por gallinaza y guano de ganado (vacuno caprino, ovino) en proporciones iguales. El primer abono se realizó los días 15 y 16 de febrero del 2008 (200 gramos a cada planta), colocándose alrededor de la planta, a una distancia de $10 \mathrm{~cm}$. En el mes de enero y marzo del año 2009, se aplicó abono por segunda y tercera vez (470 gramos a cada planta), en un radio de $15 \mathrm{~cm}$ alrededor de la planta.

Los riegos en época de sequía se realizaron con el agua de los canales el Auxilio para el predio del Número Ocho (acarreo en bidones y en acémila, gráfico 6) y el agua del canal de Aranmarca para el

Figura 6: Acarreo de agua con acémilas

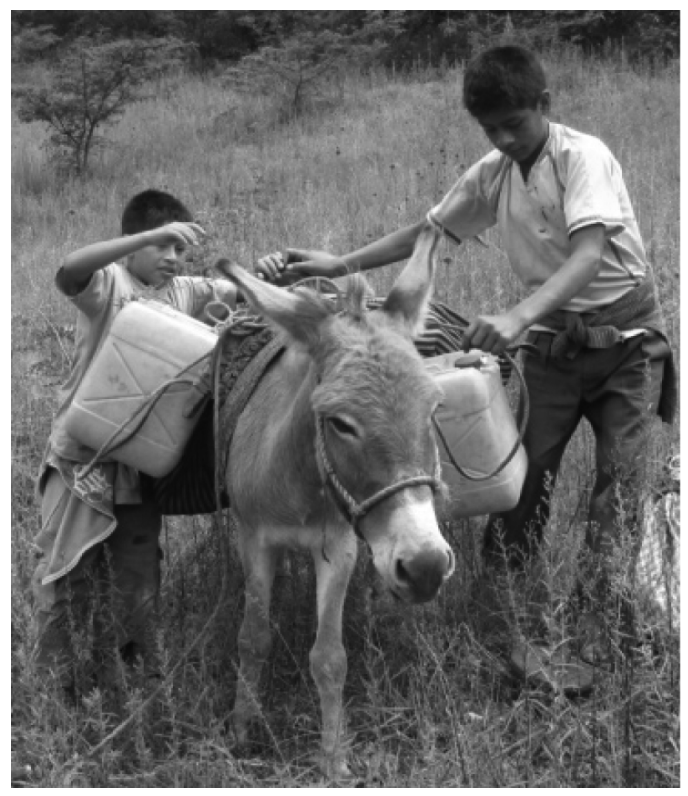

Fuente: Elaboración propia.
Figura 7: Precipitaciones promedio (mm), año 2007, microcuenca de San Juan.

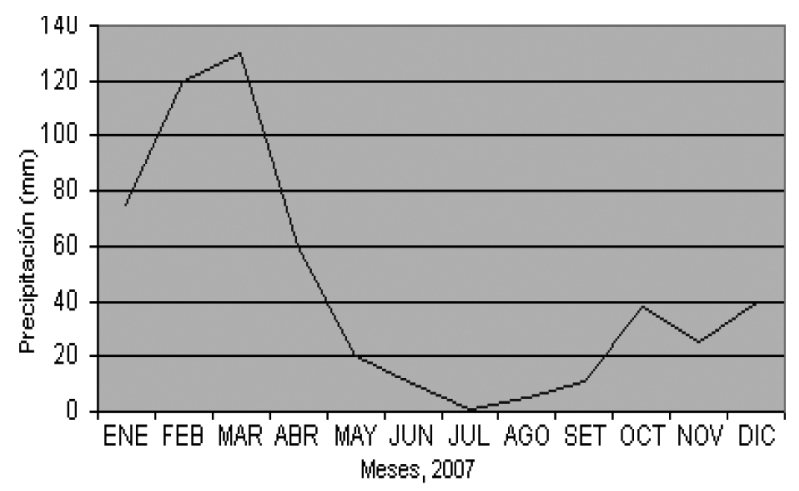

Fuente: http://www.condesan.org/cuencasandinas/jequetepeque.htm

predio de Tayopampa (riego a gravedad por inundación). El primer riego fue realizado del 5 al 10 de enero del 2008, a toda la plantación, después se realizaron riegos cada 40 días a la plantación del predio el Número ocho y cada 30 días al predio de Tayopampa (si 2 días antes del riego programado llovía, ya no se regaba).

Como datos referenciales del año 2008, se tiene que la estación de lluvias es de diciembre a marzo (lluvias 15 días por mes, precipitación 100 mm, temperatura $7-14^{\circ} \mathrm{C}$ ), la estación seca en los meses de mayo a setiembre se caracterizada por un sol moderado y templado por las noches (lluvias 2 por mes, precipitación $10 \mathrm{~mm}$, temperatura $4-22$ ${ }^{\circ} \mathrm{C}$ ). En el gráfico 7 , se presenta las precipitaciones mensuales promedio (mm) del año 2007 en la microcuenca de San Juan.

La poda (para dar forma a la copa) se realizó en el mes de enero de 2009 , se podaron a 47 plantones.

Las hojas de las plantas de tara sufrieron el ataque nocturno de la hormiga arriera (corta las hojas retrasando el desarrollo de la planta), se trató de combatir con lechada de ceniza y cal (se roció en el camino de las hormigas y en los hormigueros), al persistir se usó productos químicos como el malathion, formidor y hormix. (Cantidad: 2008, $6 \mathrm{~kg}$ de tifón y $1 / 2$ litro de malathion; el 2009, $6 \mathrm{~kg}$ de formidor y hormix y 1 litro de malathion); el malathion se aplicó anualmente por medio de un fumigador-pulverizador en la proporción de $450 \mathrm{ml}$ de malathion para 50 litros de agua, a cada uno de los predios forestados con la tara, lográndose controlar las plagas de insectos y enfermedades. 
Para disminuir la destrucción de plantones de tara debido a los animales (ganado vacuno y caprino) y transeúntes, a fines del mes de diciembre de 2008, los predios fueron cercados en su perímetro con plantas de maguey y cerco de alambre con púas.

En el cuadro 11, se presenta la comparación de crecimiento de los plantones.

Cuadro 11. Comparación del crecimiento de los plantones

\begin{tabular}{|l|c|c|c|c|c|}
\hline \multirow{2}{*}{ Predios } & $\begin{array}{c}\text { Siem- } \\
\text { bra }\end{array}$ & \multicolumn{2}{|c|}{$\begin{array}{c}\text { Manejo año } \\
\mathbf{2 0 0 8}\end{array}$} & \multicolumn{2}{|c|}{$\begin{array}{c}\text { Manejo año } \\
\mathbf{2 0 0 9}\end{array}$} \\
\cline { 2 - 6 } & $\begin{array}{c}\text { enero } \\
\mathbf{2 0 0 8}\end{array}$ & mayo & $\begin{array}{c}\text { octu- } \\
\text { bre }\end{array}$ & enero & $\begin{array}{c}\text { diciem- } \\
\text { bre }\end{array}$ \\
\hline Número Ocho & $20,0 \mathrm{~cm}$ & $23,6 \mathrm{~cm}$ & $36,0 \mathrm{~cm}$ & $81,0 \mathrm{~cm}$ & $119,0 \mathrm{~cm}$ \\
\hline Tayopampa & $16,8 \mathrm{~cm}$ & $19,0 \mathrm{~cm}$ & $27,0 \mathrm{~cm}$ & $66,0 \mathrm{~cm}$ & $95,0 \mathrm{~cm}$ \\
\hline
\end{tabular}

Fuente: Elaboración propia.

En la figura 8 , se muestra el crecimiento mensual de la plantación de tara en los 2 predios, durante los años de manejo 2008 y 2009.

Figura 8: Crecimiento de la tara durante los años 2008 y 2009

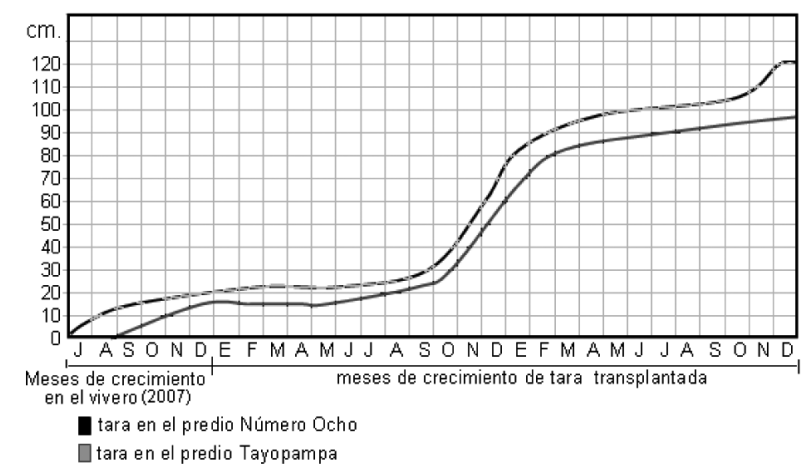

Fuente: Elaboración propia.

El menor crecimiento de los plantones del predio con riego (Toyopampa) se debió al exceso de lluvia causado por las inundaciones en los hoyos y también puede haber influido el transplante realizado antes de obtener el tamaño recomendado. ("investigaciones del cultivo de la tara" http://www.geocities.com/lebr7/tara.htm)

En el gráfico 9, se muestra una de las plantas de tara del predio el Número Ocho (secano), altura promedio de $118 \mathrm{~cm}$ (diciembre de 2009) que está en flor y con fruto (3 vainas).
Figura 9: Planta de tara predio Número Ocho

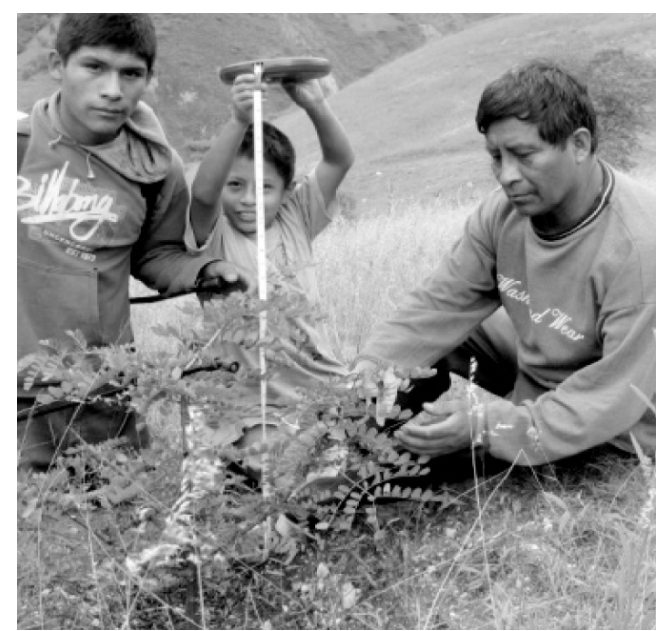

Fuente: Elaboración propia

En la figura 10, se muestra una de las plantas de tara del predio Tayopampa (riego), están en flor siendo la altura promedio de $95 \mathrm{~cm}$ (diciembre 2009).

Figura 10: Plantas de tara predio Tayopampa

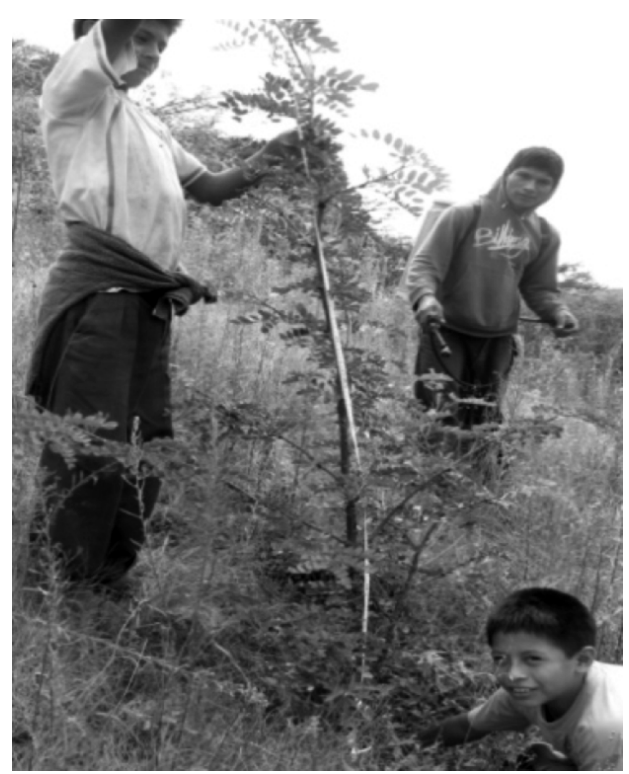

Fuente: Elaboración propia.

El proyecto comenzó con una plantación de 1546 plantones, en un área de 2 hectáreas, de acuerdo al manejo agrícola la producción inicial será después de 3 año y medio, y la producción comercial a partir del cuarto año. La producción estimada de cada árbol dado en kilogramos se hizo tomando promedios de los estudios realizados (Universidad La Molina, Instituto Internacional de productores de tara y del consultor forestal Carlos Villanueva M.). En el cuadro 12, se presenta una estimación de la producción bruta de la tara. 
Cuadro 12: Producción de tara del Proyecto Piloto (área, 2 ha)

\begin{tabular}{|c|c|c|c|c|c|c|}
\hline \multirow[b]{2}{*}{ DESCRIPCIÓN } & \multirow{10}{*}{ 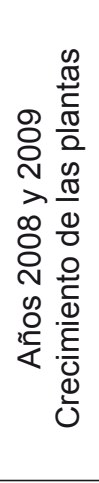 } & \multicolumn{5}{|c|}{ AÑOS PRODUCTIVOS } \\
\hline & & $\begin{array}{c}2010 \\
\text { Año } 3 \\
\end{array}$ & $\begin{array}{c}2011 \\
\text { Año } 4\end{array}$ & $\begin{array}{c}2012 \\
\text { Año } 5 \\
\end{array}$ & $\begin{array}{c}2013 \\
\text { Año } 6\end{array}$ & $\begin{array}{r}2014 \\
\text { Año } 7 \\
\end{array}$ \\
\hline Plantación realizada & & 1546 & 1546 & 1546 & 1546 & 1546 \\
\hline Plantas muertas en $\%$ & & $22 \%$ & $20 \%$ & $20 \%$ & $20 \%$ & 00 \\
\hline Plantas resembradas & & 343 & 69 & 14 & 3 & 00 \\
\hline Plantas en producción & & 1203 & 1477 & 1532 & 1543 & 1546 \\
\hline Producción kg./árbol & & 1 & 4 & 9 & 14 & 19 \\
\hline Producción de plantas aptas & & 1203 & 5908 & 13788 & 21602 & 29374 \\
\hline Cosechas $\mathrm{x}$ año & & 1 & 2 & 2 & 2 & 2 \\
\hline Producción de la plantación: año/Kg. & & 1203 & 11816 & 27576 & 43204 & 58748 \\
\hline
\end{tabular}

Fuente: Producción promedios de UNALM, Villanueva M. Carlos y acopiadores de Cajamarca

En el cuadro 13, se presenta la inversión realizada en la plantación piloto de la tara, la inversión total fue de S/. 12 234. En el cuadro 14, se presentan los bienes tangibles que suman S/. 7236 .

Cuadro 13: Inversión en la plantación piloto de la tara al 2008

\begin{tabular}{|l|lc|}
\hline \multicolumn{1}{|c|}{ CONCEPTO } & \multicolumn{2}{c|}{ SUBTOTAL } \\
\hline INVERSIÓN FIJA EN INFRAESTRUCTURA & & \\
\hline Terreno & 1000 & 2896 \\
Estanques & 3340 & \\
\hline EQUIPO, HERRAMIENTAS Y ENSERES & & \\
\hline Equipo & 680 & \\
Herramientas de labranza & 890 & \\
Herramientas de corte & 560 & \\
Herramientas de arrastre & 180 & \\
Enseres de plástico & 441 & \\
Enseres de uso diverso & 145 & \\
\hline Total & & \\
\hline
\end{tabular}

Fuente: Elaboración propia

Cuadro 14: Bienes tangibles para la forestación piloto de la tara

\begin{tabular}{|l|c|c|}
\hline TANGIBLES & & 7236 \\
\hline Inversión fija en infraestructura & 4340 & \\
\hline Equipo, herramientas y enseres & 2896 & 400 \\
\hline INTANGILES & & 3608 \\
\hline CAPITAL DE TRABAJO & & 1100 \\
\hline I. COSTOS DIRECTOS & & \\
\hline A: INSUMOS & 988 & \\
\hline Adquisición de plantones & 110 & \\
\hline Fertilizantes & & \\
\hline B: MANO DE OBRA & 340 & 280 \\
\hline Preparación de terrenos & 920 & 690 \\
\hline Hacer hoyos & 760 & S/. 12234 \\
\hline Siembra de plantones & 208 & \\
\hline Resiembra de plantones & & \\
\hline C. OTROS GASTOS & & \\
\hline II. COSTOS INDIRECTOS & & \\
\hline TOTALES & & \\
\hline
\end{tabular}

Fuentes: Elaboración propia. 
En el cuadro 15, se presenta la inversión intangible usado en el proyecto. En el cuadro 16, se presenta los gastos realizados en el proyecto en los años 2008 y 2009.

En el cuadro 17, se presenta la venta estimada de la producción de tara considerando un precio de venta referencial de S/.1,94 nuevos soles por kilo de vaina de tara. De este cuadro, se puede deducir que el año 2011 se recuperará toda la inversión. A partir del año 2014 hacia adelante, los ingresos serán más o menos constantes.

Cuadro 15: Inversión intangible usada en el proyecto

\begin{tabular}{|l|c|}
\hline ACTIVOS INTANGIBLES & Monto en soles \\
\hline . Selección de predios & 300 \\
. Definir plantación & 200 \\
. Elección de plantones & 130 \\
. Formalizar plantación & 130 \\
. Imprevistos: $5 \%$ & 40 \\
\hline Total & S/. $\mathbf{8 0 0}$ \\
\hline
\end{tabular}

Fuente: Elaboración propia.

Cuadro 16: Gastos en el proyecto de reforestación

\begin{tabular}{|l|c|c|}
\hline Descripción & Año 2008 & Año 2009 \\
\hline Gastos administrativos & 600 & 600 \\
\hline Gastos de capacitación & 200 & 200 \\
\hline Gastos operativos & 1600 & 1600 \\
\hline $\begin{array}{l}\text { Mantenimiento de ace- } \\
\text { quias }\end{array}$ & 80 & 80 \\
\hline $\begin{array}{l}\text { Mano de obra no califi- } \\
\text { cada }\end{array}$ & 720 & 720 \\
\hline Insumos & 216 & 791 \\
\hline Otros servicios & 1284 & 1305 \\
\hline Total & 4700 & 5296 \\
\hline
\end{tabular}

\section{CONCLUSIONES Y RECOMENDACIONES}

El proyecto piloto se está realizando con una inversión de S/.12 234 y durante los 2 años de manejo agrícola se han invertido S/. 9996, toda la inversión se podrá recuperar en el año 2011.

Aprovechando que los arbustos de tara están pequeños, dentro del mismo terreno, se pueden sembrar cultivos menores como la lechuga y el culantro.

En el año 2010, la municipalidad de San Juan está impulsando la forestación con tara, 10 campesinos vecinos están forestando con tara.

En temporada de sequía, el agua en el caserío de Cachilgon-Calani es abastecido por manantiales, los pobladores no tienen cultura de usar el agua en forma adecuada, el riego que utilizan es por inundación.

Por los resultados, se puede adelantar que para el crecimiento de la tara no se requiere mucha agua comparada con otras plantas del lugar, pues las plantas en el terreno secano se encuentran en mejor estado que las plantadas en zona de riego.

\section{REFERENCIAS BIBLIOGRÁFICAS}

1. Espinoza E. (1988). Cultivos andinos, $1^{\text {ra }}$. Ed., Lima, Perú

2. Montoya E., Figueroa G. (1991). Geografía de Cajamarca Vols. III y IV, 1'ra. Ed., Edit. Offset Kemoy, Lima, Perú.

3. Rodríguez A. (1983). El cultivo del níspero y el valle del Algar-Guadalest, Edita sociedad cooperativa de crédito de Callosa de Ensarriá; Alicante, España.

Cuadro 17: Venta estimada de la producción de tara ( $\mathrm{kg} / \mathrm{planta}$ al año)

\begin{tabular}{|c|c|c|c|c|c|c|}
\hline $\begin{array}{c}\text { Años } \\
\text { productivos }\end{array}$ & $\begin{array}{c}\text { Plantación en } \\
\text { producción }\end{array}$ & $\mathbf{k g} /$ planta & $\begin{array}{c}\text { Cosechas } \\
\text { por año }\end{array}$ & $\begin{array}{c}\text { Total } \\
\text { en kg }\end{array}$ & PV & $\begin{array}{c}\text { Total Bruto } \\
\text { S/. }\end{array}$ \\
\hline 2008 & 00 & 00 & 00 & 00 & 00 & 00 \\
\hline 2009 & 00 & 00 & 00 & 00 & 00 & 00 \\
\hline 2010 & 1203 & 1 & 1 & 1203 & 1,94 & 2333,82 \\
\hline 2011 & 1477 & 4 & 2 & 11816 & 1,94 & 22923,04 \\
\hline 2012 & 1532 & 9 & 2 & 27576 & 1,94 & 53497,44 \\
\hline 2013 & 1543 & 14 & 2 & 43204 & 1,94 & 83815,76 \\
\hline 2014 & 1546 & 19 & 2 & 58748 & 1,94 & 113971,12 \\
\hline
\end{tabular}

Fuente: Elaboración propia. 
4. Villanueva C. (2007). La tara. El oro verde de los Incas, 1. ${ }^{\text {ra }}$ ed., Edit. Agrum, UNALM, Lima, Perú.

5. Municipalidad distrital de San Juan (2004). Plan estratégico de desarrollo, San Juan al 2015, San Juan - Cajamarca, Perú.

6. Rojas O., Rojas N. y Díaz P. (2007). La tara y condiciones de reforestación en el Alto Jequetepeque, Microcuenca de San Juan-Cajamarca, Industrial Data: Vol. 10, N. 2, 2007, UNMSM; Lima, Perú.

7. Perú forestal en números (2007) en una economía libre de mercado http://www.sinia.minam. gob.pe/admElemento.php?accion=bajar\&idDoc Adjunto=107

8. Monografías.com (2006). Proyecto de pre factibilidad para la instalación de 100 hectáreas de tara (caesalpinia spinosa) en Jayanca Lambayeque? Perú http://www.monografias.com/tra- bajos47/plantacion-de-tara/plantacion-de-tara2. shtml (visitado el 30/12/2009)

10. Agrodata Perú (2009). Exportacion de la tara http://agrodataperu.blogspot.com/search/label/tara20\%en\%20polvo\%20PerA ${ }^{\circ}$ (visitado el 14/02/2010)

11. Metas quinquenales de plantaciones http://www.fondebosque.org.pe

12.Málaga-Webb \& Asociados (2004). PERX: Plan estratégico regional exportador de Cajamarca http://www.regioncajamarca.gob.pe/descargas/ deseco/05_09_01_PLAN_ESTRATEGICO_ REGIONAL_EXPORTADOR.pdf (Visitado el 06/10/2009)

13. Todo sobre la Tara http://www.geocities.com/lebr7/tara.htm (visita do: 18/01/2009) 